\title{
What we need to know, where we need to go
}

\section{Lines of research in aging and in EURAPA}

\author{
Heinz Mechling
}

Published online: 23 February 2013

(C) European Group for Research into Elderly and Physical Activity (EGREPA) 2013

EURAPA - State of the art: In 2010, the boards of EGREPA, EURAPA (as the Official Journal of the European Group for Research into Elderly and Physical Activity), and Springer Publishers agreed not only to publish review papers but also accept original research papers. Opening with volume 8, number 1, 2011, EURAPA announced also to accept new manuscript categories [6]. Since its early beginning, the scope of EURAPA has been defined by two lines of research: biomedical and behavioral sciences. Thus, research papers have not been limited to specific scientific disciplines. The different and still highly diversifying areas of research in aging are welcomed in EURAPA. With the inclusion of original papers, this idea was accepted by versatile scientific disciplines. This openness for many perspectives in aging research and the change in publication policy has led to an increase in papers submitted. The range of contributions has spread from different approaches in basic to a variety of perspectives in applied sciences. In order to concentrate and strengthen the broad range of perspectives, the Editors-in-Chief have additionally initiated special issues which seem to be of great importance and allow fostering topics underrepresented in the papers submitted to or already published in EURAPA. Special issues focusing on new topics will be continued and also be supported in the future. The editorial board welcomes proposals for new topics and applications for guest editorships for future issues.

Special issues: We invite colleagues active in basic science to deal not only with the aging brain-cognitive, perceptual, and energetic stimulations [5, 7] — but also with extended new topics as epigenetics and environmental influences on aging, embodiment and aging, and neurophysiological (neuroimaging), physiological, and biomechanical

H. Mechling $(\bowtie)$

Institute of Movement and Sport Gerontology, German Sport University Cologne, 50933 Cologne, Germany

e-mail: mechling@dshs-koeln.de (especially assessment and long-term monitoring of physical activity) research methods.

Colleagues from behavioral and applied sciences are invited for topics and act as guest editors dealing with test and measurement, and diagnostic instruments: development of new as well as evaluation, improvement, and adaptation of existing tests. This applies also to questionnaires related to subjective well-being, quality of life, and physical activity data if we regard their use in specific groups such as demented people, physically handicapped, and fallendangered people. A fall-related diagnostic paper has just been put online. The humanities are urgently expected to contribute. They should offer their knowledge on methods "how to reach different generations and target groups" based on psychological, pedagogical, "geragogical," and social knowledge.

Results from basic and applied research should help to overcome still existing deficits in our knowledge on the "dose-response" relationship of physical activity for different situations and different target groups and samples. A synopsis from an interdisciplinary point of view and the will of scientific disciplines to cooperate will in the long term contribute to arriving at useful conclusions and will thus lead to efficient intervention programs.

Special issue 1: The first special issue from the behavioral sciences gave attention to the "Socio-economic patterns of sport demand and aging" [3]. It focused on the determinants of physical activity and sport participation, the effects of sport expenditure, and economic determinants. For behavioral considerations and sociopolitical decisions, this perspective is strongly related to questions of how to increase participation of elderly in physical activity and sport. Questions of how to communicate and distribute new and already existing programs are considered. Answers to the influence of environmental conditions, space, and facilities for physical activities of the elderly with their socioeconomic relevance are taken into account. 
Special issue 2: The available special issue (January 2013, volume 10, issue number 1) in biomedical sciences addresses the topic of "Physical activity in oncology" [1]. This issue highlights the necessity for interdisciplinary cooperation with disciplinary advanced knowledge and methods. Basic research and behavioral, practice-oriented considerations must be brought together for a successful acceptance and implementation of disease-specific physical activities in clinical and therapeutical situations. Cancer is regarded as a part of different comorbidities in the older adults. During the last years, various positive therapeutic effects of physical activity in cancer patients have been shown and positive adaptive reactions have been measured. Different authors contributing to this issue refer to the clinical treatment of cancer, to different forms of cancer, and the relationship between cancer and physical activity therapy. They also refer to underlying mechanisms and to the historical development within 30 years of physical activity in oncology. Some papers take a broader view by integrating the topic into environment and epigenetics. Clinical trials to study the effects in various forms of cancer even for reaching individual differentiation in treatment, to define therapeutic standards, and to indicate and monitor physical exercise programs in oncology are regarded as future challenges and desirability. The objectives and demands formulated by the guest editors are "to make physical activity programs accessible to as many cancer patients as possible" and that "the role of physical activity in the prevention of cancer should be taken closer into the focus of interest."

Where we are to go: Longevity and the proportion of aged populations are increasing all over the world. This is mainly due to the progress in medicine, the control of infections, and the preventive advice on degenerative diseases associated with age. But it is not only sufficient to cure diseases, we also must face and accept the increasing necessity to prevent them. This makes prevention-because of its social and economical relevance-a top issue on the agenda of the twenty-first century. In addition to medical treatment and nutrition, physical activity is one of the most important factors in prevention. In each society, effective prevention starts at early childhood and extends up to the highest age. In this context, preventive measures for all generations do not need to rely exclusively on physical activity. Neither can prevention only be based exclusively on a medical approach, but it must also take into account psychic phenomena and effects, in particular life events and their consequences, as well as social and environmental factors. Prevention must be based on the behavior of each individual and his/her reactions when faced with one or several risk factors. We should strive for an interdisciplinary scientific and social project especially for the elderly. Nowadays, in western societies, more attention is being paid to the quality of life than to the length of life. From the point of view of basic and applied research, it should be made clear what the influencing factors are that affect the aging process. Age as such is an inevitable and insufficient predictor. Until now, genetic risk factors are regarded as non-influenceable. Therefore, basic research in the field of aging has a responsibility to advance the knowledge on aging processes and their underlying mechanisms. This expected progress in knowledge could contribute to implement intervention programs tailored for specific groups such as cancer or demented patients and settings such as in health care or sports therapy. The presently available knowledge of the physiology of the body and the length of life is shown only to depend upon genetics. The causative mechanism of aging still remains unknown. More attention should be paid to the maintenance and repair of the progressive impairment of all cellular functions that cause damage to the body. We should take a much closer look at the factors which can be influenced to prevent age-related diseases and stress our contribution to render more precisely recommendations for a turnaround in the direction of health-enhancing behaviors in physical activity and nutrition. These measures will not cure epidemic diseases in the elderly population, but they might slow down their negative development. It is obvious that the elderly are at a higher risk for deterioration of mental functioning. Therefore, special attention is to be directed to the demography-based development of dementia. Physical exercise and fitness have been proposed as potential factors that promote healthy cognitive aging. The specific hypothesis that physical exercise is a cause of healthy cognitive aging has yet to be validated [4]. Even the limitations in significant results in research papers should not hinder us from developing population- and deficit-specific physical activity and fitness programs up to the highest age.

Prevention: As far as our aging societies are concerned, prevention is the topic which should unite basic and applied-behavioral science to give meaning to our data and results. This should lead to better evaluated preventive measures for individuals and specific training groups. Thus, we will better succeed in convincing and advising our decision makers and assist them in improving and financing effective actions. The relevance of prevention is widely accepted in western societies and their politics. But it seems not to be understood that an increase of the financial basis for preventive measures is indispensable. Even in social groups that should know better as for example medical and health-care organizations or insurance companies, "prevention" seems to have a highly conflicting potential. This conflict is exemplified best in the percentage of prevention in the total health-care costs in the USA and Germany. In the USA, the total costs are 2.3 trillion US dollars; in Germany, 287 billion euros. The 
percentage for prevention is $1.5-3 \%$ in Germany and 2-3\% in the USA. The 2012 US Institute of Medicine report asserts "that 75 percent of our health care costs are related to preventable conditions. To adequately meet our prevention needs and to control our unsustainable growth in health care costs we recommend that we increase federal funding for public health and prevention by $\$ 12$ billion" (http://www.apha.org/NR/ rdonlyres/D1708E46-07E9-43E7-AB99-94A29437E4AF/0/ PrevPubHealth2012_web.pdf and http://www.forumgesundheitspolitik.de/artikel/artikel.pl?artikel=0200). What might the total of health-care costs in less developed countries be and what is their per mille fraction for prevention?

EURAPA News: In the last editorial (volume 9, issue number 2, 2012) [2], we informed that the society boards of EURAPA and EGREPA (as the Official Journal of the European Group for Research into Elderly and Physical Activity) have appointed Dr. Wiebren Zijlstra as the Editor-in-Chief for the biomedical section of EURAPA. Dr. Zijlstra, who recently moved from the University of Groningen (The Netherlands) to the German Sport University Cologne (Germany), gratefully agreed to join and has already started in his new role.

Introduction-Dr.Wiebren Zijlstra: After graduating in Human Movement Sciences at the University of Groningen, Dr. Zijlstra worked as a researcher at the Department of Clinical Neurology and Neurophysiology of the University of Freiburg, as well as at the Department of Neurology of the University of Groningen. In 1997, he received a PhD at the Medical Faculty of the University of Groningen. Following subsequent research and teaching positions, he was an associate professor at the Center for Human Movement Sciences of the University Medical Center Groningen. In June 2012, Dr. Zijlstra was appointed university professor at the German Sport University Cologne, where he now is head of the Institute of Movement and Sport Gerontology.

In his research and teaching, Dr. Zijlstra addresses physical activity and its relationships with physical and cognitive functioning in older people. His specific research interests include balance and mobility, neuromechanical adaptability, and interventions to improve health span. His research activities have included biomechanical, neurophysiological, and cognitive aspects of mobility; changes in mobilityrelated activities associated with aging or age-related diseases; and the development and evaluation of intervention approaches. The major aim of his research and teaching activities is to contribute to furthering the knowledge and methodological tools which are required for the development of effective interventions which support independent functioning in older people.

As the 'new' Editor-in-Chief for the biomedical section of EURAPA, Dr. Zijlstra fully endorses EURAPA's broad scope related to physical activity and aging in biomedical as well as in behavioral sciences. Its multi- and interdisciplinary character allows EURAPA to serve as an important platform for all researchers and practitioners who are interested in understanding the relationships between physical activity patterns and physiological, psychological, and social aspects of aging, as well as the huge impact physical activity may have on pathophysiological processes and overall functioning.

It is his hope and expectation that some recent developments will allow EURAPA to further expand its role as an interdisciplinary platform for the development of scientific knowledge relating to aging and physical activity. First of all, though EURAPA's original focus on review articles gives rise to a condense presentation and discussion of the outcomes of relevant published age-related studies, the recent inclusion of new manuscript categories (see Ref. [6]), such as original research papers, brief reports, and commentaries, allows EURAPA to include the results of different types of research activities and, hence, expands its scope of scientific discussion. Secondly, the fact that since 2010 EURAPA has been assigned an impact factor (in 2011, 0.947) has increased the visibility and attractiveness of EURAPA as a target journal for publishing. Thirdly, the open access option for publishing in EURAPA allows authors to comply with open access policies and, hence, have all advantages of open access publication such as easy and free access for all readers. Though this option has been used sparingly in all recent volumes, it is hoped that it will be used more in the near future.

It is expected that the aforementioned recent developments are among the factors that will contribute to attracting larger author- and readerships for EURAPA as the Official Journal of the European Group for Research into Elderly and Physical Activity. However, other major factors are to maintain high scientific standards, as well as an innovative content, of publications in EURAPA. For these latter factors, the continuing support of the scientific community (readers, reviewers, as well as authors) is needed. It is our hope that with your support, EURAPA will further evolve as a crucial platform for the interdisciplinary scientific exchange that is needed to understand the complex relationships between physical activity patterns and aging.

As hitherto, in the first issue of the New Year, the following EURAPA reviewers in 2012 are acknowledged:

Maxime Billot, Canada

Cornelius Bollheimer, Germany

Eling D. de Bruin, Switzerland

Niamh Maria Cummins, Ireland

Robert Van Deursen, UK

Jerri Edwards, USA

Markus Gruber, Germany

Barbara Jefferis, UK

Leigh Hale, New Zealand 
Juha Hijmans, Netherlands

Maria Machado, Portugal

Joaquim Gabriel Mendes, Portugal

KJ Mickle, Australia

Tobias Morat, Germany

Jennifer Muhaidat, UK

Samuel R. Nyman, UK

Douglas Paddon-Jones, USA

Giuseppe Pichierri, Switzerland

D. Schoene, Australia

Martin Stevens, Netherlands

N. Theill, Switzerland

Ulrike S. Trampisch, Germany

Manuel Vaquero, USA

Peter van Vliet, Netherlands

G Wu, USA

Conflict of interest None.

\section{References}

1. Baumann FT, Bloch W (in press) Physical activity in oncology. Eur Rev Aging Phy Act 10(1)

2. Brach M (2012) Editorial. Eur Rev Aging Phy Act 9(2):85

3. Breuer $\mathrm{C}$ et al (2010) Socio-economic patterns of sport demand and aging. Eur Rev Aging Phy Act 7(2):61-70

4. Etnier JL (2009) Physical activity: programming to promote cognitive function: Are we ready for prescription? In: Chodzko-Zaijko W, Kramer AF, Poon LW (eds) Enhancing cognitive functioning and brain plasticity. Champaign, Human Kinetics, pp 159-176

5. Kramer AF, Erickson KI (2007) Capitalizing on cortical plasticity: influence of physical activity on cognition and brain function. Trends Cognit Sci 11(8):342-348

6. Mechling H (2010) Five years of cooperation: EURAPA and Springer publishers - state of the art, changes and future development. Eur Rev Aging Phy Act 7(2):59-60

7. Miller et al (2012) Measuring the impact of exercise on cognitive aging: methodological issues. Neurobiol Aging 33(3):2943 